\title{
Insula Demonstrates a Non-Linear Response to Varying Demand for Cognitive Control and Weaker Resting Connectivity With the Executive Control Network in Smokers
}

\author{
John R Fedota*,', Allison L Matous', Betty Jo Salmeron', Hong Gu', Thomas J Ross' and Elliot A Stein' \\ 'Neuroimaging Research Branch, National Institute on Drug Abuse, Intramural Research Program, National Institutes of Health, Baltimore, \\ MD, USA
}

\begin{abstract}
Deficits in cognitive control processes are a primary characteristic of nicotine addiction. However, while network-based connectivity measures of dysfunction have frequently been observed, empirical evidence of task-based dysfunction in these processes has been inconsistent. Here, in a sample of smokers $(n=35)$ and non-smokers $(n=21)$, a previously validated parametric flanker task is employed to characterize addiction-related alterations in responses to varying (ie, high, intermediate, and low) demands for cognitive control. This approach yields a demand-response curve that aims to characterize potential non-linear responses to increased demand for control, including insensitivities or lags in fully activating the cognitive control network. We further used task-based differences in activation between groups as seeds for resting-state analysis of network dysfunction in an effort to more closely link prior inconsistencies in task-related activation with evidence of impaired network connectivity in smokers. For both smokers and non-smokers, neuroimaging results showed similar increases in activation in brain areas associated with cognitive control. However, reduced activation in right insula was seen only in smokers and only when processing intermediate demand for cognitive control. Further, in smokers, this task-modulated right insula showed weaker functional connectivity with the superior frontal gyrus, a component of the task-positive executive control network. These results demonstrate that the neural instantiation of salience attribution in smokers is both more effortful to fully activate and has more difficulty communicating with the exogenous, task-positive, executive control network. Together, these findings further articulate the cognitive control dysfunction associated with smoking and illustrate a specific brain circuit potentially responsible.

Neuropsychopharmacology (20 I6) 4I, 2557-2565; doi: I 0.I038/npp.20 I6.62; published online 25 May 2016
\end{abstract}

\section{INTRODUCTION}

Cognitive control refers to the adaptive changes enacted to perform goal-directed thoughts and actions (Mars et al, 2011). In addition to the central role of cognitive control in goal-directed behavior, deficits in these processes are a primary characteristic of nicotine-and other drug-addiction (Goldstein and Volkow, 2011; Kalivas and Volkow, 2005; Volkow et al, 2016). To this point, prominent theories of addiction posit that deficits in prefrontal cortical function (Goldstein and Volkow, 2011) and impaired cognitive control (Volkow et al, 2016) may contribute to substance abuse via both an inability to inhibit prepotent behaviors related to drug seeking (Lubman et al, 2004) as well as the biased processing of interoceptive signals associated with drug craving and withdrawal (Paulus and Stewart, 2014).

\footnotetext{
*Correspondence: Dr JR Fedota, Neuroimaging Research Branch, National Institute on Drug Abuse, Intramural Research Program, National Institutes of Health, 25I Bayview Boulevard, Baltimore, MD 21224, USA, Tel: + | 443740 2782, Fax: + I 443740 2753,

E-mail: john.fedota@nih.gov

Received 9 February 2016; revised 7 April 2016; accepted 7 April 2016; accepted article preview online 26 April 2016
}

Both of these functional deficits may manifest as impaired engagement of prefrontal cortex during effortful control processing.

The neuroanatomical foci of cognitive control processing are distributed bilaterally throughout the brain, including the insulae and dorsolateral prefrontal, lateral parietal, and medial frontal cortices (Nee et al, 2007). It is notable that many of these regions are also hubs within large-scale brain networks, including the executive control network (ECN), associated with task-positive activation (Fox et al, 2005), and the salience network (SN), linked to conscious and subconscious salience attribution (Seeley et al, 2007). The SN and ECN, along with the default mode network (DMN), associated with planning, ruminations, and mind wandering (Raichle et al, 2001), encompass a tripartite system implicated in attentional allocation (Kelly et al, 2008).

Despite the confluence of cognitive control process and network hub localization, empirical evidence of specific cognitive control dysfunction in smokers has been inconsistent. Behavioral and neurobiological deficits of inhibitory processing are often dissociated in smokers (Buzzell et al, 2014; de Ruiter et al, 2012; Evans et al, 2009), and consistent effects on performance monitoring have also proven elusive 
(Franken et al, 2010; Luijten et al, 2011; Rass et al, 2014). These disparate task-based results are in contrast to resting network connectivity, which is more consistently dysregulated in smokers (for a review, see Fedota and Stein, 2015; Sutherland et al, 2012). Resting functional connectivity results show that connectivity within and between the ECN, SN, and DMN is disrupted, with SN-ECN connectivity reduced and $\mathrm{SN}-\mathrm{DMN}$ connectivity increased in smokers, especially during abstinence (Lerman et al, 2014; Sutherland et al, 2013).

Recent evidence suggests that the application of cognitive control is aversive (Botvinick, 2007; Inzlicht et al, 2015) and the activation of control processes is necessarily subject to an internal cost-benefit analysis (Kool et al, 2013). Thus the activation of cognitive control resources is unlikely to be a binary, all-or-nothing series of computational processes. Instead, efficient activation of control processes is predicted to scale as demands for control increase. That said, the lion's share of investigations into cognitive control dysfunction in addiction rely on discrete comparisons of the presence $v s$ absence of control demands. Such binary comparisons may lead to the inconsistencies in the empirical findings described above, as they only characterize the top and bottom of the functional range of cognitive control processing.

One way to better characterize the full range of cognitive control deficits in smokers is to examine the variable recruitment of control resources across an array of demands (ie, high, medium, and low). Such an approach has the benefit of characterizing potential non-linear responses to increased demand for control, including insensitivities or lags in fully activating the cognitive control network. This is in addition to the ability to characterize differences in overall activation associated with cognitive control as seen in discrete presence/absence paradigms. Thus a range of demands for control, with its high ecological validity, provides a doseresponse relationship (both in response magnitude and variable temporal resource engagement) between the demand for control and its associated neurobiological response.

Although task paradigms that parametrically manipulate demand for control have been employed in healthy controls (Fan et al, 2014; Forster et al, 2011; Wendt et al, 2014), such paradigms have rarely been employed in addiction research. Prior evidence of interactions between working memory load and smoking state (sated, abstinent) has been observed in smokers, where abstinence leads to increased activation in dorsal lateral prefrontal cortex (dlPFC) at low, but not medium or high, working memory load (Xu et al, 2005). However, this analysis was limited to a small cohort of smokers $(n=9)$ and did not address differences between smokers and non-smokers.

The current study employed the parametric flanker task (PFT) - a previously validated paradigm shown to modulate electrophysiological indices of cognitive control in healthy individuals (Forster et al, 2011) - to interrogate potential differences in cognitive control mechanisms recruited in the face of parametrically increasing environmental demands. The PFT is employed in an effort to fully describe the demand-response curve for cognitive control processing in smokers as compared with non-smokers.

Our second goal was to explore the underlying neurocircuitry supporting any observed smoking-related processing dysfunctions. This is based in part on recent successes integrating task-based activation with resting network analyses to provide a more holistic understanding of addiction-related dysfunction (Janes et al, 2015; Sutherland et al, 2013). Predicated on the known nicotine-dependent alterations between and among preestablished, large-scale brain networks (Fedota and Stein, 2015; Sutherland et al, 2012), our strategy was to use PFT-based differences in activation between smokers and non-smokers as seeds for resting-state functional connectivity ( $\mathrm{rsFC}$ ) analysis. Thus the current study sought to more directly relate tasked-based dysfunction in cognitive control to large-scale network connectivity by identifying differences in network connections between task-modified, cognitive control regions and resting-state networks relevant to attentional salience attribution.

\section{MATERIALS AND METHODS}

\section{Participants}

Participants were required to be right-handed, aged 18-55 years, free of active drug or alcohol abuse/dependence (other than nicotine dependence in smokers), reporting no current psychiatric or neurological disorders, and presenting no contraindications for magnetic resonance (MR) imaging. Fifty-eight participants (36 smokers and 22 non-smoking controls) completed all experimental procedures. Following preprocessing, data from one smoker and one non-smoker were discarded owing to excessive head motion (see Supplementary Table S1 for full demographic profile). Written informed consent was obtained in accordance with the National Institute on Drug Abuse, Intramural Research Program Institutional Review Board.

Upon arrival for their MR scanning session, participants underwent a medical assessment, including a urine test for recent use of illicit drugs (opiates, oxycodone, benzodiazepines, buprenorphine, cocaine, amphetamines/methamphetamines, tetrahydrocannabinol (THC), methadone, phencyclidine, and methlenedioxymethamphetamine) and alcohol (Breathalyzer). Positive urine tests were exclusionary for all drugs except THC. For THC, positive urine tests were followed by the Drug Evaluation and Classification neuromotor exam to determine whether the participant was intoxicated (Heishman et al, 1996). Positive neuromotor exams were exclusionary. Smokers were instructed to smoke ad lib prior to MR scanning; the last cigarette was smoked an average of $48.6 \mathrm{~min}(\mathrm{SEM}=7.69)$ before MR scanning.

\section{Experimental Design}

Participants completed an 8-min, eyes open resting scan directly followed by the PFT. This task order was maintained across all participants in an effort to collect unbiased restingstate data prior to any task performance. All stimuli were presented using the E-Prime software (Psychology Software Tools, Sharpsburg, PA), and behavioral responses were collected with an MR-compatible button box.

As previously described (Forster et al, 2011), the PFT is a modified version of the classic Eriksen flanker task designed to instantiate varying levels of demand for cognitive control on a trial-by-trial basis via response conflict (Figure 1). The PFT stimulus array consists of seven white letters (' $\mathrm{S}$ ' and/or ' $\mathrm{H}$ ') 


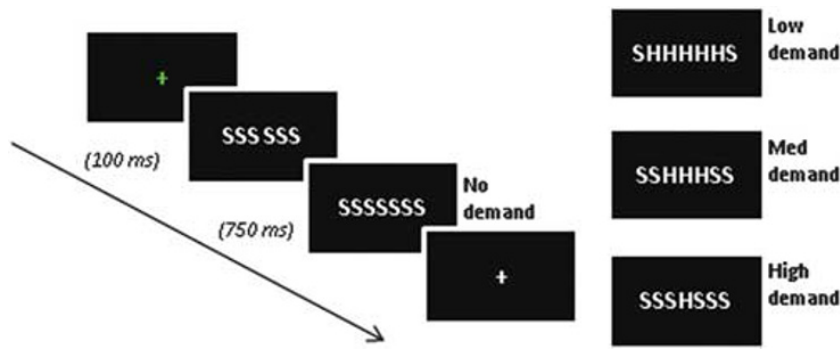

Figure I Task paradigm. Demand for control is parametrically modulated via the number of conflicting stimuli flanking the target stimulus at the center of the array. Flankers appear on screen $100 \mathrm{~ms}$ prior to target stimuli. Participants responded with left/right index finger mapped to target $(H / S)$ and were instructed to equally prioritize speed and accuracy.

displayed horizontally across the center of a black screen. Participants were instructed to respond as quickly and accurately as possible with either their right or left index finger (counterbalanced across participants) corresponding to the target letter in the middle of the string. During noconflict/demand trials, all flankers matched the target letter (ie, SSSSSSS, HHHHHHH). During high-conflict/demand trials the target letter differed from all flankers $\mathrm{HHHSHHH}$, SSSHSSS). In medium-conflict/demand trials, the target and adjacent flankers were identical, but the outermost flankers differed (HHSSSHH, SSHHHSS). During low-conflict/demand trials, the target was identical to all but the two outermost flankers (HSSSSSH, SHHHHHS).

Two hundred and forty randomly ordered trials grouped into five $5-\mathrm{min}$ runs (25 min total) were presented. Trials were split 50/50 between compatible and demand trials (trial totals: 120 compatible, and 40 each of high, medium, and low demand). Flanker stimuli were presented $100 \mathrm{~ms}$ prior to target stimuli and target+flankers remained on the screen for $750 \mathrm{~ms}$. Inter-trial interval was jittered from 500 to $7000 \mathrm{~ms}$ in a Poisson distribution to improve event-related fMRI modeling.

\section{MRI Data Acquisition}

Whole-brain echo planar images were acquired on a 3T Siemens Trio scanner (Erlangen, Germany) using a 12-channel head coil. For both resting-state and PFT data, 39 oblique axial slices (4-mm thick; $30^{\circ}$ to anterior commissure-posterior commissure line) were acquired using a T2*-weighted, single-shot gradient echo, echo planar imaging sequence sensitive to blood oxygenation level-dependent (BOLD) effects (720 volumes (PFT); 241 volumes (resting); repetition time $(\mathrm{TR})=2000 \mathrm{~ms}$; echo time $(\mathrm{TE})=27 \mathrm{~ms}$; flip angle $(\mathrm{FA})=78^{\circ}$; field of view $220 \times 220 \mathrm{~mm}^{2}$; image matrix $64 \times 64)$. High-resolution oblique-axial T1-weighted structural images were also acquired using a 3D magnetization-prepared rapid gradient-echo (MPRAGE) sequence (TR $=1900 \mathrm{~ms}$; $\mathrm{TE}=3.51 \mathrm{~ms} ; \mathrm{TI}=900 \mathrm{~ms} ; \mathrm{FA}=9^{\circ}$; voxel size $\left.=1 \mathrm{~mm}^{3}\right)$.

\section{Behavioral Analysis}

Behavioral effects of GROUP (smoker/non-smoker) and demand for cognitive control, DEMAND (High/Medium/ Low), were quantified via correct response time (RT) and trial accuracy using $\mathrm{R}$ Project for Statistical Computing (package afex, function mixed (Singmann et al, 2015)). In addition, based on the parametric task manipulation of interest, a priori contrasts of GROUP effects at each level of DEMAND were performed with Bonferroni corrections for multiple comparisons.

RT data were submitted to a linear mixed model with a random intercept per subject and fixed effects of GROUP and DEMAND. Accuracy data were submitted to a generalized linear mixed model with a binomial distribution and logit link function with a random intercept per subject and fixed effects of GROUP and DEMAND. A model with random intercepts for subject and slopes for DEMAND and fixed effects of GROUP and DEMAND failed to converge owing to the high accuracy and subsequent reduced variance in low and medium DEMAND conditions across both groups.

\section{MRI Analysis}

\section{Parametric flanker task}

Preprocessing. PFT BOLD data were processed in AFNI (https://afni.nimh.nih.gov/afni). Data were slice-time and motion-corrected and aligned with anatomical images via non-linear registration. Following motion correction, motion censoring was performed on any two consecutive time points with Euclidean distance derivative values $>0.3 \mathrm{~mm}$. Time series were normalized to the percentage of signal change and spatially smoothed to an $8-\mathrm{mm}$ full-width at halfmaximum (Friedman et al, 2006).

Single-subject analysis. PFT data were submitted to a voxel-wise multiple regression analysis with regressors expressed as a delta function convolved with a standard hemodynamic response function and its temporal derivative. Regressors included stimulus type (compatible, high demand, medium demand, and low demand) for both correct and error responses (eight total regressors) as well as six head motion parameters. In addition, contrasts of (demand correct)-(compatible correct) responses were calculated for high, medium, and low demand responses individually. A voxel-wise average amplitude change equal to the percentage change from baseline $(\beta)$ was calculated per participant and regressor.

Group analysis. Based on a previous meta-analysis of cognitive control network activation across a variety of tasks (Nee et al, 2007), MNI coordinates of regions in anterior cingulate cortex (ACC), precuneus, and bilateral dlPFC, insula, and inferior parietal lobule were identified and combined into a single mask. Eight-mm radius spheres were centered on these coordinates, and the resulting mask (Supplementary Figure S1) was used as a small volume correction (SVC) for PFT BOLD analysis.

Voxel-wise thresholds corrected for multiple comparisons were calculated using Monte Carlo simulations. Significance was determined as meeting or exceeding minimum cluster extent criteria at $p$-corrected $<0.05$. The direction of significant results was confirmed with corrected $(p<0.05)$ contrasts. PFT BOLD data were analyzed using multivariate modeling in AFNI via 3dMVM (Chen et al, 2013) for the full GROUP by DEMAND (High-Compatible/Med-Compatible/ 

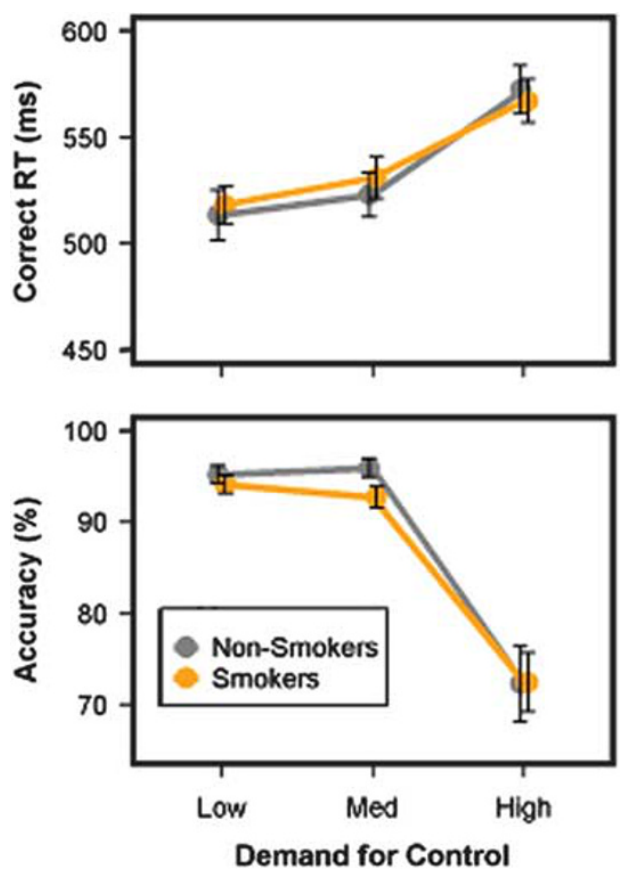

Figure 2 Behavioral results. Correct response time (RT) increased monotonically as demand for control increased in both smokers (orange) and non-smokers (gray). Accuracy decreased as demand for control increased for both groups. A GROUP $\times$ DEMAND interaction was observed for accuracy. Follow-up contrasts showed a trend-level $(p=0.05)$ group effect (Smokers $<$ Non-smokers) for processing medium demand for control. Error bars reflect SEM.

Low-Compatible) model, and mixed-effects multilevel analysis via 3dMEMA (Chen et al, 2012) for a priori contrasts of GROUP effects at each of the three levels of DEMAND (High-Compatible/Med-Compatible/Low-Compatible). These a priori contrasts were performed with Bonferroni corrections for multiple comparisons ( $p$-voxel $<0.0167, p$-corrected $<0.0167)$. In addition, an exploratory whole-brain analysis for the GROUP by DEMAND model was performed ( $p$-voxel $<0.001$, p-corrected $<0.05)$.

\section{Resting-State Functional Connectivity}

Preprocessing. Resting BOLD data were analyzed using AFNI and SPM12 (http://www.fil.ion.ucl.ac.uk/spm/). Data were slice-time and motion-corrected, quadratically detrended, and spatially normalized to MNI space via nonlinear registration, followed by temporal band-pass filtering $(0.01-0.1 \mathrm{~Hz})$. Individual white matter (WM), and cerebrospinal fluid (CSF) masks were obtained by anatomical image segmentation in SPM and were used to extract signals from non-neuronal sources. Nuisance covariates, including six-motion parameters and signals in the first four principal components of WM/CSF were regressed out, followed by Gaussian spatial smoothing (full-width at halfmaximum $=8 \mathrm{~mm}$ ).

Single-subject analysis. For each subject, correlation coefficient (CC) images were computed by correlating each voxel's motion-censored time course with a reference time course from a seed defined by GROUP effects as described above. Resulting subject-level CC images were Fisher's $Z$-transformed ( $Z$-images) to better approximate a normal distribution.

Group analysis. Identified GROUP differences in task activation were used as seeds for rsFC analysis. Analyses were a priori constrained to voxels falling within each of the three anatomical masks of large-scale brain networks consistently shown relevant to nicotine addiction (Fedota and Stein, 2015; Sutherland et al, 2012): DMN, ECN, and SN. These resting networks were identified via group-level independent component analysis (gICA) (Beckmann et al, 2005) within the FSL software package MELODIC (http:// www.fmrib.ox.ac.uk/fsl/melodic/index.html). The number of components was set to 20. Between-subject analysis and group statistical comparison were conducted using the dualregression approach. First, individual component time courses were derived from regressing the individual resting-state BOLD data against the gICA component maps. Second, the variance-normalized time series from the above spatial regressions were voxel-wise correlated with the corresponding resting-state fMRI time courses to estimate the individual component maps. Results from all participants were then subject to one sample $t$-test against zero. A threshold of $t>5.6$ with a corrected $p<0.05$ (uncorrected $p<5 \times 10^{-5}$ ) was applied to generate final maps for each gICA component. Components of DMN, ECN, and SN were identified via visual inspection. For each gICA-identified network, an individual mask of resultant voxels (Supplementary Figure S2) was used in analysis of seedbased rsFC (see below).

$T$-tests of GROUP difference in rsFC between a task-based seed and voxels within a given network mask were performed (seed-ECN voxels; seed-SN voxels; seed-DMN voxels), with Bonferroni correction for multiple comparisons applied ( $p$-voxel $<0.0167$, $p$-corrected $<0.0167)$. In addition, an exploratory unmasked whole-brain rsFC analysis with the task-based seed was performed for GROUP $(p$-voxel $<0.001, p$-corrected $<0.05)$.

\section{RESULTS}

\section{Parametric Flanker Task}

Behavioral. A main effect of DEMAND was observed for correct RT, $\mathrm{F}(2,5574.80)=72.71, p<0.001$. Follow-up contrasts showed that RT increased from low/medium to high levels of DEMAND (Figure 2, Supplementary Table S2). A priori tests for GROUP effects at each level of DEMAND were nonsignificant.

In contrast, a GROUP $\times$ DEMAND interaction was observed for response accuracy, $X^{2}(2)=7.89, \quad p=0.02$. Follow-up tests of GROUP effects at each level of DEMAND revealed a trend effect at medium DEMAND, $X^{2}(1)=3.78$, $p=0.05$, with smokers compared with non-smokers showing lower accuracy. Similar GROUP differences were not observed at either high or low DEMAND (Figure 2).

fMRI. A main effect of DEMAND was observed across multiple nodes of the a priori defined cognitive control network. These effects were observed in bilateral insulae, $\mathrm{L}$ precentral gyrus, $\mathrm{R}$ middle frontal gyrus, and $\mathrm{L}$ inferior 
a

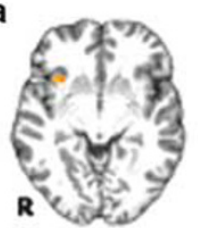

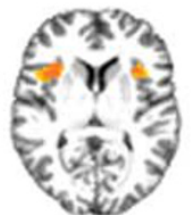
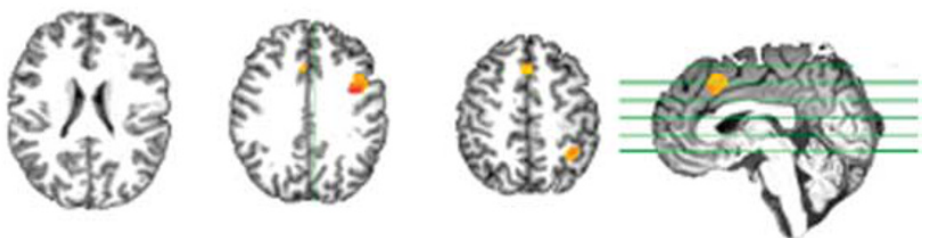

ipl
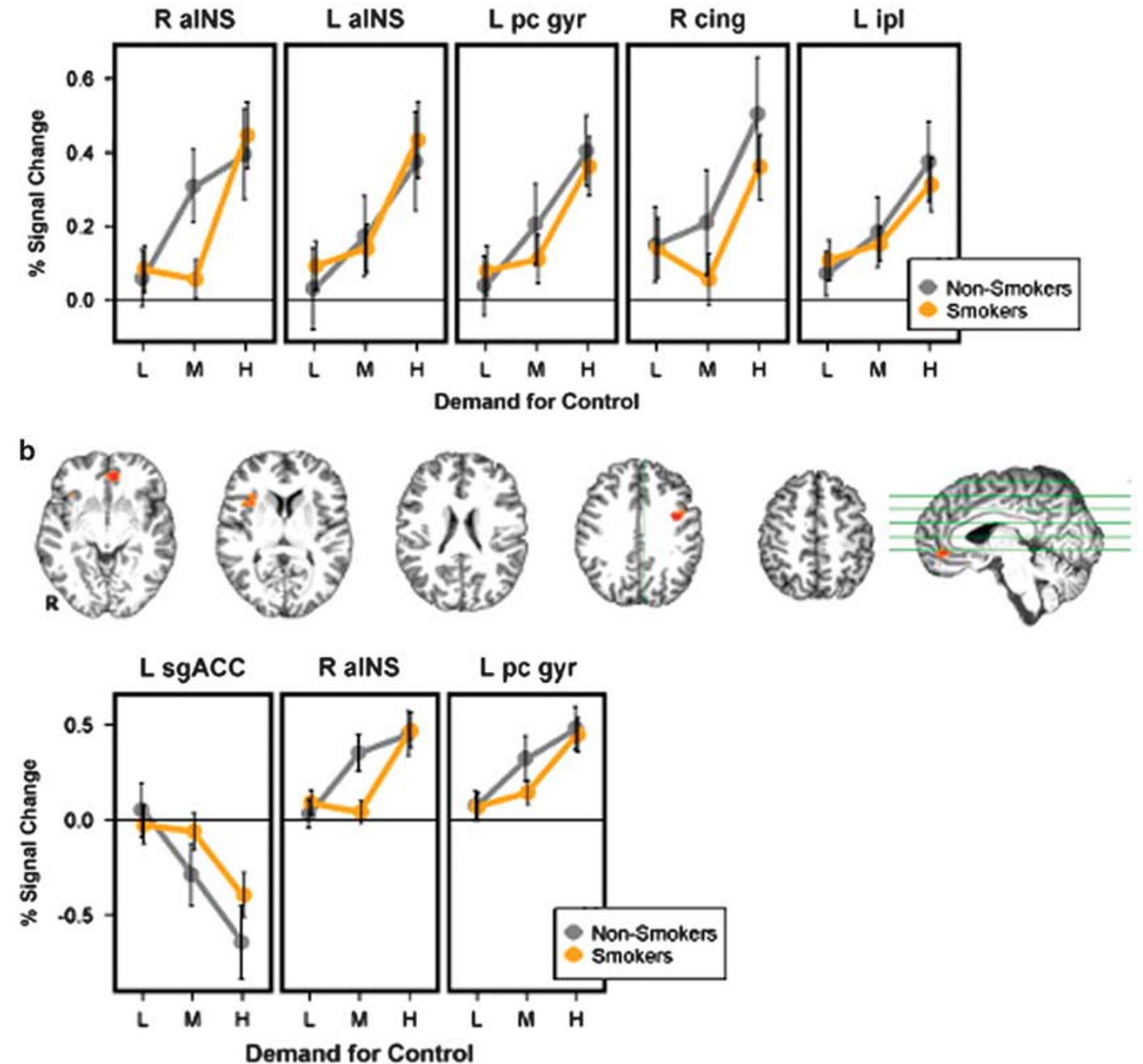

Demand for Control

Figure 3 Main effect of demand for control. In both smokers (orange) and non-smokers (gray), a main effect of DEMAND was observed. (a) Within the ROls of the cognitive control network (Supplementary Figure SI), increasing demand for control led to increased activation across multiple ROls. (b) Exploratory whole-brain analysis showed similar results in R alNS and L pc gyr and additionally showed a monotonic decrease in activation in both groups in R sgACC as demand for control increased. Error bars reflect SEM. L alNS, left anterior insula; $L$ ipl, left intraparietal lobule; $L$ pc gyr, left precentral gyrus; $\mathrm{R}$ cing, right cingulate gyrus; $\mathrm{R}$ alNS, right anterior insula; $\mathrm{R}$ sgACC, right subgenual anterior cingulate cortex.

parietal lobule (Figure 3a). Across these regions, activation increased monotonically as demand for control increased. No main effect of GROUP or GROUP $\times$ DEMAND interaction was observed.

Exploratory whole-brain analysis showed a main effect of DEMAND in $\mathrm{R}$ aINS and left precentral gyrus regions that overlapped with those described in the SVC cognitive control network results. In addition, left subgenual ACC showed a main effect of DEMAND, though in this region activation decreased monotonically as demand for cognitive control increased (Figure 3b). GROUP contrasts at each level of DEMAND failed to show any significant differences in the whole-brain analysis.
Individual a priori GROUP contrasts at each level of DEMAND showed a significant reduction in right anterior insula ( $\mathrm{R}$ aINS) activation in smokers but only when processing medium DEMAND stimuli (Figure 4). Notably, this activation cluster overlapped with the right insula clusters showing a main effect of DEMAND above (Figure $3 \mathrm{a}$ and $\mathrm{b}$ ) and was qualitatively similar in both cases.

Resting-state functional connectivity. The R aINS ROI that demonstrated a GROUP effect when processing medium demand for cognitive control (Figure 4) was used as a seed in rsFC analyses restricted within each of the gICA-identified 

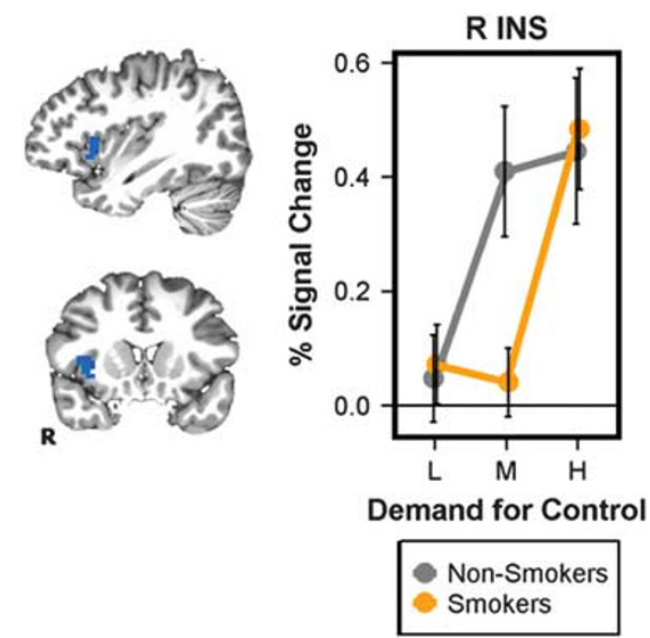

Figure 4 Group effect during processing of medium demand for control. An a priori GROUP contrast of activation across the cognitive control ROI mask during processing of medium demand for control identified a right anterior insula ( $R$ alNS) region with reduced activation in smokers. BOLD response extracted from this $\mathrm{ROI}$ during processing of low and high demand stimuli showed no GROUP differences, while the main effect of DEMAND described above (Figure 3) remains. Error bars reflect SEM.

large-scale networks relevant to addiction. A GROUP effect was only observed for rsFC between the R aINS seed and voxels within the ECN. Specifically, in smokers vs nonsmokers, weaker functional connectivity in an R aINS-right superior frontal gyrus circuit was observed (Figure 5). In contrast, no GROUP difference was seen in connectivity between the task-based R aINS seed and voxels within either the DMN or SN. Exploratory whole-brain rsFC analysis with the task-based $\mathrm{R}$ aINS seed also failed to yield significant GROUP differences.

\section{DISCUSSION}

In the current study, demand for cognitive control was parametrically modulated in an effort to more completely characterize the range of neurobiological responses to conflict and to explore potential differences in the demand-response relationship between smokers and nonsmokers. Behavioral results demonstrated that both groups were equally successful at varying their performance in response to increasing levels of demand for control, such that correct RT increased and accuracy decreased monotonically from low to medium to high demand for cognitive control. However, smokers did show a trend toward reduced accuracy compared with controls at the intermediate demand level.

In addition, a specific dysfunction in cognitive control processing was observed in smokers performing the PFT. This dysfunction was manifest as a non-linear response to increasing demand for cognitive control but only in the $\mathrm{R}$ aINS. Importantly, while $\mathrm{R}$ aINS in smokers was insensitive to intermediate demands for control, it did not show insensitivity to all levels of conflict. Activation of $\mathrm{R}$ aINS to high demand scaled appropriately and was indistinguishable between smokers and non-smokers. Thus
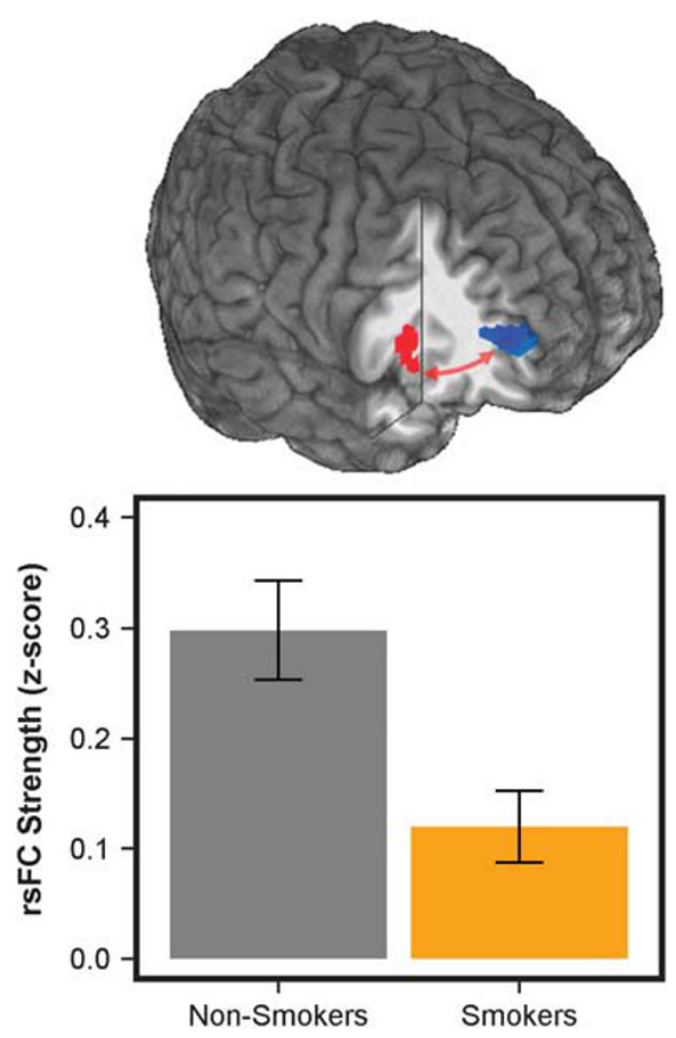

Figure 5 Resting-state functional connectivity ( $r s F C$ ) effects. Using the right anterior insula (R alNS) ROI identified in task-based analysis (Figure 4) as a seed (red), resting-state connectivity with the gICA identified that executive control network (ECN) (Supplementary Figure S2) was weaker in smokers as compared with non-smokers. Specifically, connectivity between $\mathrm{R}$ alNS and voxels within the right superior frontal gyrus (blue) was weaker in smokers, independent of parametric flanker task activation. Error bars reflect SEM.

smokers' deficit in cognitive control processing is not an absolute loss of function but instead what appears to be a focal increase in the threshold of demand necessary to activate the $\mathrm{R}$ aINS. That is, in smokers $\mathrm{R}$ aINS is slow to reach full activation and does so only when processing high demands for control. Importantly, this non-linear response pattern was not observed in other nodes of the previously well-established cognitive control network-including left insula, left parietal cortex, and medial frontal gyrus-each of which showed similar monotonic changes in activation with increasing demands for cognitive control in both smokers and non-smokers. Thus the majority of cognitive controlrelated brain regions appear to be unaffected by smoking, at least in performance of the PFT. The commonality in activation between smokers and non-smokers across control processing regions outside of R aINS may be another reason for the inconsistency in prior task-based findings of control deficits in smokers (Buzzell et al, 2014; de Ruiter et al, 2012; Evans et al, 2009; Franken et al, 2010; Luijten et al, 2011; Rass et al, 2014).

The localization of the observed non-linear response to demand for cognitive control within R aINS is notable, as prior evidence implicates the insula in the attribution of salience to internal and external events and stimuli (Chang et al, 2013; Cieslik et al, 2015; Craig, 2009; Klein et al, 2013). This purported insular function is in line with the prefrontal 
dysfunction in salience attribution theorized to be compromised in addiction (Goldstein and Volkow, 2011); the present rsFC findings strengthen this interpretation.

Resting functional connectivity between this R aINS foci and right superior frontal gyrus (R SFG) is lower in smokers as compared with non-smokers. In smokers, this weakened functional connectivity strength is notable as both the seed ( $\mathrm{R}$ aINS) and target ( $\mathrm{R}$ SFG) are nodes within large-scale brain networks relevant to addiction (Fedota and Stein, 2015; Sutherland et al, 2012) and attentional control (Seeley et al, 2007; Sridharan et al, 2008). The insula is a primary node of the SN, while R SFG is a primary component of the ECN. In nicotine addiction, it has been theorized (Sutherland et al, 2012) and subsequently experimentally demonstrated (Lerman et al, 2014) that connectivity between SN and ECN is impaired and that the allocation of attention to salient internal or external cues is correspondingly dysfunctional (Droutman et al, 2015; Naqvi et al, 2014). The weaker connectivity strength between $\mathrm{R}$ aINS and $\mathrm{R}$ SFG is consistent with evidence of an impaired ability in smokers to efficiently direct attention exogenously (Claus et al, 2013; Hahn et al, 2007) and is in agreement with prior neuroimaging evidence of structural changes in each of these nodes tied to chronic nicotine exposure. Smokers have reduced gray matter volume in SFG (Brody et al, 2004) as well as a negative correlation between cigarette exposure (pack-years) and insular thickness (Morales et al, 2014). In addition, gray matter density is decreased in both SFG (Brody et al, 2004; Zhang et al, 2011a) and the insula (Gallinat et al, 2006; Stoeckel et al, 2015) for smokers as compared with non-smokers. However, increased left insular gray matter density was reported in smokers as compared with non-smokers and is associated with the Toronto Alexithymia Scale total score and the difficulty-identifyingfeelings factor (Zhang et al, 2011a).

We propose that the observed non-linear response in $\mathrm{R}$ aINS increases the threshold at which otherwise adequately processed demands for control are integrated into an identification of salient stimuli. Such a shift in activation threshold is in line with prior evidence of an abstinenceinduced increase in dlPFC activation in smokers when preforming a low load working memory task (Xu et al, 2005). The present results suggest that, for smokers, the neural instantiation of salience attribution in R aINS is both harder to fully activate and perhaps in turn has more difficulty communicating with the exogenous facing, task-positive, ECN. An ostensible cause for the observed decrease in $\mathrm{R}$ aINS activation in response to intermediate demand is the primacy of interoceptive processing in drug abuse (Paulus and Stewart, 2014). In smokers, focus on endogenous signals of nicotine craving and/or withdrawal may inappropriately absorb the insular capacity otherwise available in healthy non-smokers for orientation toward salient, exogenous stimuli.

The current results inform the apparent paradox of insula activation in smokers, where hypoactivation is seen in cognitive control tasks (reviewed in Droutman et al, 2015), while hyperactivation is seen in response to smoking cues (Claus et al, 2013; Franklin et al, 2007; Janes et al, 2010; Zhang et al, 2011b). Previous results have described preferential processing of drug cues as an attenuated threshold for processing endogenous signals associated with craving (Paulus and Stewart, 2014). Here we describe an increased threshold within the cognitive control network of smokers when non-drug stimuli are processed. This suggests a misattribution of salience (in this case, its absence) that is directionally consistent with the idea that smokers underweight salience of non-drug-related stimuli via an increased threshold to recruit cognitive control resources.

Importantly, there is a non-linear relationship in this underweighting of non-smoking cues by smokers. When demands for control are large, the cognitive control network-including $\mathrm{R}$ aINS - is able to process these demands in a manner indistinguishable from non-smokers. Thus, with strong enough environmental demand for control, any attentional bias away from non-smoking stimuli is fully compensated. This suggests that deficits in processing demand for cognitive control in smokers do not involve the top of the functional dynamic range. It is not that smokers are unable to achieve a sufficient level of $\mathrm{R}$ aINS activation in response to high demands for control. Instead, they are slow to fully recruit these cognitive resources and do so only when greatly taxed. Thus the SN, partially instantiated in $\mathrm{R}$ aINS, is not deactivated in smokers; it is instead biased away from exogenous stimuli. The inclusion of an intermediate demand for control in the current paradigm provided the vehicle to develop this novel conjecture.

The observed non-linear relationship between $\mathrm{R}$ aINS activation and demand for cognitive control in smokers may further refine the role of insular function in smoking addiction. Stroke lesion data indicate that an incapacitated insula is advantageous for smoking cessation (Naqvi et al, 2007). These prior findings are seemingly antithetical to observations of reduced insular activation in smokers in the present study and elsewhere (for a review, see Droutman et al, 2015; Naqvi et al, 2014). However, recent reframing of the role of insular deactivation (Naqvi et al, 2014) suggests that functional segmentation of the insula allows for a more nuanced description of this structure's role in both attentional control and nicotine addiction. Beyond the described dysfunction of R aINS, other insular subregions (ie, ventral anterior, posterior) have been more strongly related to endogenous signal processing (Chang et al, 2013; Klein et al, 2013). Further, deactivation of these insular subregions may be vital to the disruption of smoking-related behavior and the proposed imbalance in salience attribution between endogenous and exogenous stimuli. The present results are limited to the anterior insula as defined by the cognitive control mask derived from Nee et al. (2007) and show that, within this region specifically, the processing of demands for control are impaired in smokers. Although we describe concomitant dysfunction in the onset of cognitive control processing within the anterior insula, the current data cannot directly address the differential contributions of ventral or posterior insular subregions, both of which appear to make unique contributions to the maintenance of nicotine addiction (Droutman et al, 2015; Naqvi et al, 2014). Future investigations of insular thresholding and network connectivity are needed to address these questions.

The current task-based and functional connectivity findings show nicotine addiction manifests in part as an increase in the activation threshold of R aINS in smokers, leading to an inability to properly modulate attention in the face of increasing-but still submaximal-demand for cognitive 
control. This interpretation is strengthened by the presence of a downregulated circuit between the region of task dysfunction and the R SFG, a node of the ECN associated with attentional allocation. A less efficient connection between these network nodes may be associated with inefficient executive control of attention, especially in situations of salience misattribution. Together these findings further articulate the nature of cognitive control dysfunction associated with nicotine dependence and illustrate a specific brain circuit potentially responsible. These selective differences in the processing of demand for cognitive control in the PFT provide a mechanistic description of a specific deficit in smokers that, while not directly responsible for relapse, may instead help precipitate such events via insensitivity to situations requiring prophylactic, subthreshold levels of control.

\section{FUNDING AND DISCLOSURE}

This study was supported by the National Institute on Drug Abuse (NIDA), Intramural Research Program and FDA grant NDA13001-001-00000 to EAS. The authors declare no conflict of interest.

\section{ACKNOWLEDGMENTS}

We thank Kim Slater, Angela Neal, Kate Shire, and the NIDA-IRP nursing and recruitment staff for their assistance with data collection. We also thank Sarah Forster for providing experimental stimuli and presentation code.

\section{REFERENCES}

Beckmann CF, DeLuca M, Devlin JT, Smith SM (2005). Investigations into resting-state connectivity using independent component analysis. Philos Trans Roy Soc Lond B Biol Sci 360: 1001-1013.

Botvinick MM (2007). Conflict monitoring and decision making: reconciling two perspectives on anterior cingulate function. Cogn Affect Behav Neurosci 7: 356-366.

Brody AL, Mandelkern MA, Jarvik ME, Lee GS, Smith EC, Huang JC et al (2004). Differences between smokers and nonsmokers in regional gray matter volumes and densities. Biol Psychiatry 55: 77-84.

Buzzell GA, Fedota JR, Roberts DM, McDonald CG (2014). The N2 ERP component as an index of impaired cognitive control in smokers. Neurosci Lett 563: 61-65.

Chang LJ, Yarkoni T, Khaw MW, Sanfey AG (2013). Decoding the role of the insula in human cognition: functional parcellation and large-scale reverse inference. Cereb Cortex 23: 739-749.

Chen G, Saad ZS, Britton JC, Pine DS, Cox RW (2013). Linear mixed-effects modeling approach to FMRI group analysis. Neuroimage 73: 176-190.

Chen G, Saad ZS, Nath AR, Beauchamp MS, Cox RW (2012). FMRI group analysis combining effect estimates and their variances. Neuroimage 60: 747-765.

Cieslik EC, Mueller VI, Eickhoff CR, Langner R, Eickhoff SB (2015). Three key regions for supervisory attentional control: evidence from neuroimaging meta-analyses. Neurosci Biobehav Rev 48: 22-34.

Claus ED, Blaine SK, Filbey FM, Mayer AR, Hutchison KE (2013). Association between nicotine dependence severity, BOLD response to smoking cues, and functional connectivity. Neuropsychopharmacology 38: 2363-2372.
Craig ADB (2009). How do you feel-now? The anterior insula and human awareness. Nat Rev Neurosci 10: 59-70.

de Ruiter MB, Oosterlaan J, Veltman DJ, van den Brink W, Goudriaan AE (2012). Similar hyporesponsiveness of the dorsomedial prefrontal cortex in problem gamblers and heavy smokers during an inhibitory control task. Drug Alcohol Depend 121: 81-89.

Droutman V, Read SJ, Bechara A (2015). Revisiting the role of the insula in addiction. Trends Cogn Sci 19: 414-420.

Evans DE, Park JY, Maxfield N, Drobes DJ (2009). Neurocognitive variation in smoking behavior and withdrawal: genetic and affective moderators. Genes Brain Behav 8: 86-96.

Fan J, Van Dam NT, Gu X, Liu X, Wang H, Tang CY et al (2014). Quantitative characterization of functional anatomical contributions to cognitive control under uncertainty. J Cogn Neurosci 26: 1490-1506.

Fedota JR, Stein EA (2015). Resting-state functional connectivity and nicotine addiction: prospects for biomarker development. Ann NY Acad Sci 1349: 64-82.

Forster SE, Carter CS, Cohen JD, Cho RY (2011). Parametric manipulation of the conflict signal and control-state adaptation. J Cogn Neurosci 23: 923-935.

Fox MD, Snyder AZ, Vincent JL, Corbetta M, Van Essen DC, Raichle ME (2005). The human brain is intrinsically organized into dynamic, anticorrelated functional networks. Proc Natl Acad Sci USA 102: 9673-9678.

Franken IHA, van Strien JW, Kuijpers I (2010). Evidence for a deficit in the salience attribution to errors in smokers. Drug Alcohol Depend 106: 181-185.

Franklin TR, Wang Z, Wang J, Sciortino N, Harper D, Li Y et al (2007). Limbic activation to cigarette smoking cues independent of nicotine withdrawal: a perfusion fMRI study. Neuropsychopharmacology 32: 2301-2309.

Friedman L, Glover GH, Krenz D, Magnotta V (2006). Reducing inter-scanner variability of activation in a multicenter fMRI study: role of smoothness equalization. Neuroimage 32: 1656-1668.

Gallinat J, Meisenzahl E, Jacobsen LK, Kalus P, Bierbrauer J, Kienast T et al (2006). Smoking and structural brain deficits: a volumetric MR investigation. Eur J Neurosci 24: 1744-1750.

Goldstein RZ, Volkow ND (2011). Dysfunction of the prefrontal cortex in addiction: neuroimaging findings and clinical implications. Nat Rev Neurosci 12: 652-669.

Hahn B, Ross TJ, Yang Y, Kim I, Huestis MA, Stein EA (2007). Nicotine enhances visuospatial attention by deactivating areas of the resting brain default network. J Neurosci 27: 3477-3489.

Heishman SJ, Singleton EG, Crouch DJ (1996). Laboratory validation study of drug evaluation and classification program: ethanol, cocaine, and marijuana. J Anal Toxicol 20: 468-483.

Inzlicht M, Bartholow BD, Hirsh JB (2015). Emotional foundations of cognitive control. Trends Cogn Sci 19: 126-132.

Janes AC, Farmer S, Peechatka AL, Frederick B, de B, Lukas SE (2015). Insula-dorsal anterior cingulate cortex coupling is associated with enhanced brain reactivity to smoking cues. Neuropsychopharmacology 40: 1561-1568.

Janes AC, Pizzagalli DA, Richardt S, Frederick B, de B, Holmes AJ et al (2010). Neural substrates of attentional bias for smokingrelated cues: an FMRI study. Neuropsychopharmacology 35: 2339-2345.

Kalivas PW, Volkow ND (2005). The neural basis of addiction: a pathology of motivation and choice. Am J Psychiatry 162: 1403-1413.

Kelly AMC, Uddin LQ, Biswal BB, Castellanos FX, Milham MP (2008). Competition between functional brain networks mediates behavioral variability. Neuroimage 39: 527-537.

Klein TA, Ullsperger M, Danielmeier C (2013). Error awareness and the insula: links to neurological and psychiatric diseases. Front Hum Neurosci 7: 14. 
Kool W, McGuire JT, Wang GJ, Botvinick MM (2013). Neural and behavioral evidence for an intrinsic cost of self-control. PLoS One 8: e72626.

Lerman C, Gu H, Loughead J, Ruparel K, Yang Y, Stein EA (2014). Large-scale brain network coupling predicts acute nicotine abstinence effects on craving and cognitive function. JAMA Psychiatry 71: 523-530.

Lubman DI, Yücel M, Pantelis C (2004). Addiction, a condition of compulsive behaviour? Neuroimaging and neuropsychological evidence of inhibitory dysregulation. Addiction 99: 1491-1502.

Luijten M, van Meel CS, Franken IHA (2011). Diminished error processing in smokers during smoking cue exposure. Pharmacol Biochem Behav 97: 514-520.

Mars RB, Sallet J, Rushworth MFS, Yeung N (eds) (2011). Neural Basis of Motivational and Cognitive Control. MIT Press: Cambridge, MA, USA. Retrieved from https://books.google. com/books?hl = en\&lr = \&id = A_eoYgtLmFMC\&pgis $=1$.

Morales AM, Ghahremani D, Kohno M, Hellemann GS, London ED (2014). Cigarette exposure, dependence, and craving are related to insula thickness in young adult smokers. Neuropsychopharmacology 39: 1816-1822.

Naqvi NH, Gaznick N, Tranel D, Bechara A (2014). The insula: a critical neural substrate for craving and drug seeking under conflict and risk. Ann NY Acad Sci 1316: 53-70.

Naqvi NH, Rudrauf D, Damasio H, Bechara A (2007). Damage to the insula disrupts addiction to cigarette smoking. Science 315: 531-534.

Nee DE, Wager TD, Jonides J (2007). Interference resolution: insights from a meta-analysis of neuroimaging tasks. Cogn Affect Behav Neurosci 7: 1-17.

Paulus MP, Stewart JL (2014). Interoception and drug addiction. Neuropharmacology 76(Pt B): 342-350.

Raichle ME, MacLeod AM, Snyder AZ, Powers WJ, Gusnard DA, Shulman GL (2001). A default mode of brain function. Proc Natl Acad Sci USA 98: 676-682.

Rass O, Fridberg DJ, O’Donnell BF (2014). Neural correlates of performance monitoring in daily and intermittent smokers. Clin Neurophysiol 125: 1417-1426.
Seeley WW, Menon V, Schatzberg AF, Keller J, Glover GH, Kenna $\mathrm{H}$ et al (2007). Dissociable intrinsic connectivity networks for salience processing and executive control. J Neurosci 27: 2349-2356.

Singmann H, Bolker B, Westfall J (2015). afex: Analysis of Factorial Experiments. R package version 0.14-2. http://CRAN.R-project. org/package $=$ afex.

Sridharan D, Levitin DJ, Menon V (2008). A critical role for the right fronto-insular cortex in switching between central-executive and default-mode networks. Proc Natl Acad Sci USA 105: 12569-12574.

Stoeckel LE, Chai XJ, Zhang J, Whitfield-Gabrieli S, Evins AE (2015). Lower gray matter density and functional connectivity in the anterior insula in smokers compared with never smokers. Addict Biol (e-pub ahead of print; doi:10.1111/adb.12262).

Sutherland MT, Carroll AJ, Salmeron BJ, Ross TJ, Hong LE, Stein EA (2013). Down-regulation of amygdala and insula functional circuits by varenicline and nicotine in abstinent cigarette smokers. Biol Psychiatry 74: 538-546.

Sutherland MT, McHugh MJ, Pariyadath V, Stein EA (2012). Resting state functional connectivity in addiction: lessons learned and a road ahead. Neuroimage 62: 1-15.

Volkow ND, Koob GF, McLellan AT (2016). Neurobiologic advances from the brain disease model of addiction. $N$ Engl $J$ Med 374: 363-371.

Wendt M, Luna-Rodriguez A, Jacobsen T (2014). Utility-based early modulation of processing distracting stimulus information. J Neurosci 34: 16720-16725.

Xu J, Mendrek A, Cohen MS, Monterosso J, Rodriguez P, Simon SL et al (2005). Brain activity in cigarette smokers performing a working memory task: effect of smoking abstinence. Biol Psychiatry 58: 143-150.

Zhang X, Salmeron BJ, Ross TJ, Geng X, Yang Y, Stein EA (2011a). Factors underlying prefrontal and insula structural alterations in smokers. Neuroimage 54: 42-48.

Zhang X, Salmeron BJ, Ross TJ, Gu H, Geng X, Yang Y et al (2011b). Anatomical differences and network characteristics underlying smoking cue reactivity. Neuroimage 54: 131-141.

Supplementary Information accompanies the paper on the Neuropsychopharmacology website (http://www.nature.com/npp) 